punto org

Collana diretta da Luigi Maria Sicca

25 


\title{
MONDO CONTADINO E AZIONE MERIDIONALISTA
}

L'ESPERIENZA DEL GRUPPO ROSSI-DORIA A PORTICI

\author{
prefazione \\ Ernesto Mazzetti \\ postfazione \\ Franco Vitelli
}

Editoriale Scientifica

Napoli 
Tutti i diritti sono riservati

(C) 2016 Editoriale Scientifica srl

Via San Biagio dei Librai 39

80138 Napoli

www.editorialescientifica.com

info@editorialescientifica.com

ISBN 978-88-9391-010-1 


\section{Indice}

$9 \quad$ Nota editoriale di Luigi Maria Sicca

13 Prefazione di Ernesto Mazzetti

21 PREMESSA

37 1. IL FERVORE E LE DELUSIONI DEGLI ANNI '50 E SEGUENTI

57 2. Il "Gruppo di PorticI" E L'AZIONE MERIDIONALISTA

99 3. L'APPROCCIO SOCIOLOGICO E LA CIVILTÀ CONTADINA

115 4. L'APPORTO DEI COLLEGHI STRANIERI

139 5. TRE ESPERIENZE PARTICOLARI

139 5.1. Friedrich G. Friedmann e i Sassi di Matera

162 5.2. Edward C. Banfield ed il "familismo amorale"

182 5.3. Olaf F. Larson e la "Cornell Technique"

197 6. I CICLI VENTENNALI E LE LORO INFLUENZE NEGATIVE

198 5.1. Primo ventennio (1900-1919)

201 5.2. Secondo ventennio (1920-1939)

207 5.3. Terzo ventennio (1940-1959)

226 5.4. Quarto ventennio (1960-1979)

244 5.5. Quinto ventennio (1980-1999)

251 7. L'INELUDIBILE PROCESSO DELLA MODERNIZZAZIONE

263 8. Il MEZZOGIORNO TRADITO 
287 9. I RAPPORTI CITTÀ-CAMPAGNA E LO SVILUPPO COMUNITARIO

305 10. LA “RIVOLUZIONE MERIDIONALE” MANCATA

329 CONCLUSIONI

337 Postfazione di Franco Vitelli

\section{Bibliografia}

363 Indice dei nomi

373 Hanno scritto nella Collana punto org 
(...) Ma nei sentieri non si torna indietro.

Altre ali fuggiranno

dalle paglie della cova,

perché lungo il perire dei tempi

l'alba è nuova, è nuova.

(R. Scotellaro, Sempre nuova è l'alba) 


\section{Viaggio a Mezzogiorno}

puntOorg si arricchisce dello scritto di Gilberto-Antonio Marselli. Un contributo denso di esperienza accademica e di vita vissuta sul campo, a contatto diretto con protagonisti e interpreti della storia economica e politica del Mezzogiorno.

Le ragioni di questo volume e la sua genesi sono illustrate dallo stesso autore nell' introduzione: quel viaggio all'Università degli Studi di Bari; poi a Matera e alla Chiesa di San Rocco, immersi nella disarmante purezza dell'essenziale intorno a noi; il passaggio a Tricarico, nel silenzio condiviso sulla tomba di Rocco Scotellaro e tutto quanto vissuto in quei giorni trovano oggi seguito in questa nuova esperienza editoriale.

$* * *$

puntOorg, da sempre, e poi dal primo numero nel 2010, si impegna a comprendere le "categorie" che sottendono il pensiero economico-manageriale e le prassi organizzative che ne derivano. Noi pensiamo e auspichiamo che questo atteggiamento

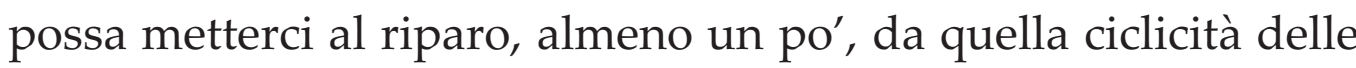
tempeste che, in modo ingenuo o sintomatico, cela invece, sovente, sempre gli stessi mali.

Ecco due esempi di categorie su cui lavorare, a partire dalle sollecitazioni che derivano dalla riflessione di Marselli.

1) La crisi. Nelle sue plurali manifestazioni, è da studiare non solo e non tanto come dato storico, situato, contingente, ma 
nella sua dimensione fenomenologica. Capire il comune denominatore che attraversa congiunture (apparentemente) molto diverse l'una dall'altra, significa disporre di una fonte di pensiero (e di vantaggio competitivo) per fronteggiare, di volta in volta, la crisi successiva, forse più grande, di certo più lunga, stante gli intervalli asintotici che accompagnano le economie occidentali dal 1929 al 2008. E il Mezzogiorno, con la sua storia di crisi (al plurale o al singolare), ha bisogno - davvero urgente - di andare meglio a fondo su tutto ciò.

2) Le risorse ${ }^{1}$. Siano quelle umane, quelle finanziarie o quelle naturali (penso, ad esempio, all'acqua e alla lezione, Dei principi di Talete), occorre cogliere il nocciolo della questione, familiarizzare con l'archetipo di riferimento. Perché agire sugli archetipi significa investire sul linguaggio, trasversale a ciò che appare, potente strumento di azione nel presente; al tempo stesso una luce da lontano sul futuro. Reazione allo sgretolio di certezze e abbondanze, vere o presunte, che l'età moderna e post, con le sue rivoluzioni, ci aveva in qualche modo consegnato ${ }^{2}$.

L'impegno è di proseguire su questa strada, perché la ricerca sulle categorie richiede ingegno, pazienza e tempo. Impone abilità ad approfondire il contatto con i contesti, da analizzare empiricamente, da un lato, e con salde e articolate strutture teoriche, dall'altro. Lo stesso Marselli sta lavorando in questi mesi a un nuovo libro, tarato su queste due polarità.

$$
* * *
$$

${ }^{1}$ Ci siamo dedicati, in precedenti numeri della Collana punto org, alle risorse umane (e all'importanza strategica delle competenze), alle risorse dell'arte (spesso non scarse), espressione di archetipi come il suono che si fa musica, o il corpo che diviene danza, il colore che si fa pittura.

2 Rinvio, nello specifico, a Iaccarino, M. (2012), Un mondo assetato. Come il bisogno di acqua plasma la civiltà (prefazione di Francesco Paolo Casavola; postfazione di Adriano Giannola), Napoli, Editoriale Scientifica. 
Come tipicamente proposto nei progetti puntOorg, l'analisi testuale è accompagnata a gesti grafici altri: in questo caso, le fotografie inserite nel volume sono un dono di Henri Cartier-Bresson a Gilberto-Antonio Marselli e da lui autorizzate per questa pubblicazione.

Nel dare alle stampe questo libro, desideriamo ringraziare Ernesto Mazzetti e Franco Vitelli, per essere intervenuti, rispettivamente, con prefazione e postfazione; Luca Carnevale per avere realizzato il disegno di copertina dalla foto scattata durante il nostro... viaggio a Mezzogiorno. 